\title{
Transarterial Therapy for Primary Liver Carcinomas: A Crack in the Armor?
}

\author{
Paul J. Karanicolas, MD, PhD, FRCS(C) $)^{1,2}$ and Yoo-Joung Ko, MD, MMSc, SM, FRCP(C) $)^{3,4}$ \\ ${ }^{1}$ Department of Surgery, The Odette Cancer Centre at Sunnybrook Health Sciences Centre, Toronto, ON, Canada; \\ ${ }^{2}$ Department of Surgery, University of Toronto, Toronto, Canada; ${ }^{3}$ Division of Medical Oncology, The Odette Cancer \\ Centre at Sunnybrook Health Sciences Centre, Toronto, ON, Canada; ${ }^{4}$ Department of Medicine, University of Toronto, \\ Toronto, Canada
}

Primary liver carcinomas are a heterogeneous group of malignancies historically classified according to the cell of origin as hepatocellular carcinoma (HCC) or intrahepatic cholgangiocarcinoma (ICC). When localized to the liver, the optimal treatment for these tumors is surgical resection (or transplantation). Unfortunately, only the minority of patients with primary liver carcinomas are candidates for these potentially curative approaches due to extrahepatic metastases, coexisting liver disease, intrahepatic satellite nodules, or locally unresectable primary tumor. ${ }^{1,2}$

Patients who are not candidates for locoregional therapy often are treated with systemic therapy. Despite improvement in systemic therapy for both of these diseases, the response rates and overall survival for patients with primary liver carcinomas remain dismal.

Two recent trials highlight the advances in systemic therapy for these diseases, and more importantly, emphasize the limitations of this approach. The SHARP Trial compared systemic sorafenib with placebo in 602 patients with advanced $\mathrm{HCC}^{3}$ The trial was stopped prematurely due to the clear benefit for patients receiving sorafenib. The median overall survival time was 10.7 months in the sorafenib group compared with 7.9 months for the patients who received placebo.

For patients with biliary tract cancers, the ABC-02 Trial demonstrated remarkably similar results. The median overall survival time was 11.7 months for patients treated with a

(C) Society of Surgical Oncology 2015

First Received: 19 June 2015;

Published Online: 31 July 2015

P. J. Karanicolas, MD, PhD, FRCS(C)

e-mail: Paul.karanicolas@sunnybrook.ca combination of gemcitabine and cisplatin compared with 8.1 months for patients who received gemcitabine alone. ${ }^{4}$

Despite the benefit of these therapies in terms of the ultimate patient-important outcome of overall survival, it must be noted that in both of these trials, the actual tumor response rate was modest at best: $26.1 \%$ of patients treated in the ABC- 02 trial and only $2 \%$ of the patients treated in the SHARP trial. These trials are clearly an important advance for medical oncologists, who now have therapies to offer patients with primary liver carcinomas that confer some benefit. Unfortunately, patients who have advanced primary liver tumors treated with systemic therapy still have little hope of long-term disease control and virtually no possibility of proceeding to curative-intent therapy.

In this issue of the Annals of Surgical Oncology, Fowler and colleagues from Washington University report their experience managing 79 patients with biphenotypic primary liver carcinoma, a rare tumor with features of both HCC and ICC. ${ }^{5}$ The patients were treated with a variety of methods including liver transplantation $(n=6)$, resection $(n=27)$, hepatic arterial therapy $(n=18)$, and systemic therapy $(n=28)$. The overall report provides valuable information about the long-term prognosis of patients with this rare disease. The most compelling finding is the identification of hepatic arterial therapies as a potentially potent treatment method for primary liver malignancies.

The patients were treated with one of three arterial therapies: transarterial chemoembolization (TACE, $n=6$ ), transarterial radioembolization (TARE, $n=6$ ), and hepatic artery infusional pump chemotherapy (HAIP, $n=6$ ). As might be expected, the treatment selected for the patients varied based on the extent of disease. The patients selected for systemic therapy were far more likely to have distant metastases at diagnosis than the patients treated 
with arterial therapies (58 vs. $12 \%$ ). This difference in baseline characteristics limits the ability to compare survival across groups. Indeed, stratification in the survival curves likely represents differences in the underlying extent of disease rather than the comparative efficacy of the therapies. The more valid comparison between systemic therapy and transarterial therapies is in the response rates.

Given the results of the two aforementioned trials, it should not be surprising that the response rates were poor for the patients who received systemic therapy, with only $6 \%$ of the patients exhibiting a partial response. In contrast, the response rate was an impressive $47 \%$ among the patients treated with arterial therapy. The best results occurred with the patients who received HAIP. Four of six patients demonstrated a response, and the remaining two patients had stable disease. Perhaps most impressively, two patients had a sufficient response to allow surgical resection of residual disease.

Transarterial therapies (TACE in particular) hold an established role in the management of locally advanced HCC, supported by two randomized controlled trials. ${ }^{6-8}$ The role of transarterial therapy in the management of ICC, particularly the impact of HAIP, is less well defined. Hepatocellular carcinoma is a hypervascular tumor, and the benefit of TACE in this setting may be more related to the ischemic effect of the embolization than to the cytotoxic effect of chemotherapy. ${ }^{9,10}$ In contrast, ICC tends to be hypovascular and less likely to benefit from embolic/ischemic therapies. Nevertheless, because ICC is frequently confined to the liver but locally unresectable, there is compelling biologic rationale for locoregional therapies.

Several small, single-center studies have examined the role of TACE, TARE, and HAIP for patients with unresectable ICC. In a pooled analysis of these studies, HAIP achieved the highest response to therapy (57\%) and the longest median overall survival (23 months). ${ }^{11}$

Several consensus statements advocate the use of HAIP for patients with liver-only colorectal metastases, where it has been extensively studied. ${ }^{12-14}$ Although the data supporting this method for primary liver cancer are more limited, the rationale may in fact be stronger because there is a greater likelihood that the disease is truly confined to the liver. The main limitation of HAIP therapy is the requirement for an operation to implant the pump, with associated morbidity.

Currently, a multicenter phase 2 trial examining the impact of HAIP on primary liver carcinomas is accruing patients. ${ }^{15}$ Given the paucity of data supporting HAIP in the context of primary liver carcinomas, surgeons should await the results of this trial before widespread adoption of this technique. Nonetheless, the data accumulating regarding the role of transarterial therapies in the management of primary liver carcinomas provides some optimism to patients and clinicians that a more effective locoregional therapy is on the horizon.
DISCLOSURE There are no conflicts of interest.

\section{REFERENCES}

1. Endo I, Gonen M, Yopp AC, et al. Intrahepatic cholangiocarcinoma: rising frequency, improved survival, and determinants of outcome after resection. Ann Surg. 2008;248:84-96.

2. El-Serag HB, Siegel AB, Davila JA, et al. Treatment and outcomes of treating of hepatocellular carcinoma among Medicare recipients in the United States: a population-based study. $J$ Hepatol. 2006;44:158-66.

3. Llovet JM, Ricci S, Mazzaferro V, et al. Sorafenib in advanced hepatocellular carcinoma. $N$ Engl J Med. 2008;359:378-90.

4. Valle J, Wasan H, Palmer DH, et al. Cisplatin plus gemcitabine versus gemcitabine for biliary tract cancer. $N$ Engl J Med. 2010;362:1273-81.

5. Fowler K, Saad NE, Brunt E, et al. Biphenotypic primary liver carcinomas: assessing outcomes of hepatic directed therapy. Ann Surg Oncol. doi:10.1245/s10434-015-4774-y.

6. Llovet JM, Real MI, Montana X, et al. Arterial embolisation or chemoembolisation versus symptomatic treatment in patients with unresectable hepatocellular carcinoma: a randomised controlled trial. Lancet. 2002;359:1734-9.

7. Lo CM, Ngan H, Tso WK, et al. Randomized controlled trial of transarterial lipiodol chemoembolization for unresectable hepatocellular carcinoma. Hepatology. 2002;35:1164-71.

8. Llovet JM, Burroughs A, Bruix J. Hepatocellular carcinoma. Lancet. 2003;362:1907-17.

9. Chang JM, Tzeng WS, Pan HB, et al. Transcatheter arterial embolization with or without cisplatin treatment of hepatocellular carcinoma: a randomized controlled study. Cancer. 1994;74: 2449-53.

10. Kawai S, Okamura J, Ogawa M, et al. Prospective and randomized clinical trial for the treatment of hepatocellular carcinoma: a comparison of lipiodol-transcatheter arterial embolization with and without adriamycin (first cooperative study). The Cooperative Study Group for Liver Cancer Treatment of Japan. Cancer Chemother Pharmacol. 1992;31(Suppl):S1-6.

11. Boehm LM, Jayakrishnan TT, Miura JT, et al. Comparative effectiveness of hepatic artery based therapies for unresectable intrahepatic cholangiocarcinoma. J Surg Oncol. 2015;111:21320.

12. Chung KY, Kemeny N. Regional and systemic chemotherapy for primary hepatobiliary cancers and for colorectal cancer metastatic to the liver. Semin Radiat Oncol. 2005;15:284-98.

13. Abdalla EK, Bauer TW, Chun YS, et al. Locoregional surgical and interventional therapies for advanced colorectal cancer liver metastases: expert consensus statements. HPB Oxford. 2013;15: 119-30.

14. Karanicolas PJ, Metrakos P, Chan K, et al. Hepatic arterial infusion pump chemotherapy in the management of colorectal liver metastases: expert consensus statement. Curr Oncol. 2014;21:e129-36.

15. Memorial Sloan Kettering Cancer Center, Ohio State University, University of Texas, Washington University School of Medicine. Hepatic arterial infusion (HAI) with floxuridine (FUDR) and dexamethasone (Dex) combined with systemic gemcitabine and oxaliplatin in patients with unresectable intrahepatic cholangiocarcinoma (ICC). In: ClinicalTrials.gov [Internet]. National Library of Medicine (US), Bethesda, MD), 2000. Retrieved 10 June 2015 https://clinicaltrials.gov/ct2/show/NCT01862315 NLM. Identifier: NCT01862315. 